\title{
General Quadratic-Additive Type Functional Equation and Its Stability
}

\author{
Yang-Hi Lee ${ }^{1}$ and Soon-Mo Jung ${ }^{2}$ \\ ${ }^{1}$ Department of Mathematics Education, Gongju National University of Education, Gongju 32553, Republic of Korea \\ ${ }^{2}$ Mathematics Section, College of Science and Technology, Hongik University, Sejong 30016, Republic of Korea
}

Correspondence should be addressed to Soon-Mo Jung; smjung@hongik.ac.kr

Received 9 December 2015; Accepted 28 February 2016

Academic Editor: Vladimir V. Mityushev

Copyright ( 2016 Y.-H. Lee and S.-M. Jung. This is an open access article distributed under the Creative Commons Attribution License, which permits unrestricted use, distribution, and reproduction in any medium, provided the original work is properly cited.

We investigate the general functional equation of the form $f(a x+b y+c z)-a b f(x+y)-b c f(y+z)-a c f(x+z)-a((a+1) / 2-$ $b-c) f(x)-b((b+1) / 2-a-c) f(y)-c((c+1) / 2-a-b) f(z)-(a(a-1) / 2) f(-x)-(b(b-1) / 2) f(-y)-(c(c-1) / 2) f(-z)=0$, whose solutions are quadratic-additive mappings in connection with stability problems.

\section{Introduction}

In 1940, Ulam [1] posed an important problem concerning the stability of group homomorphisms. In the following year, Hyers [2] solved the problem for the case of Cauchy additive functional equation. After a period longer than two decades, Rassias [3] generalized Hyers' result and then Găvruta [4] extended Rassias' result by allowing unbounded control functions. The concept of stability introduced by Rassias and Găvruta is known today with the term "generalized HyersUlam stability" of functional equations.

A solution to the functional equation

$$
f(x+y)-f(x)-f(y)=0
$$

is called an additive mapping and a solution to the functional equation

$$
f(x+y)+f(x-y)-2 f(x)-2 f(y)=0
$$

is called a quadratic mapping. If a mapping can be expressed by the sum of an additive mapping and a quadratic mapping, then we call the mapping a quadratic-additive mapping. Now, we consider the general quadratic-additive type functional equation

$$
\begin{aligned}
& f(a x+b y+c z)-a b f(x+y)-b c f(y+z) \\
& -a c f(x+z)-a\left(\frac{a+1}{2}-b-c\right) f(x) \\
& -b\left(\frac{b+1}{2}-a-c\right) f(y) \\
& -c\left(\frac{c+1}{2}-a-b\right) f(z)-\frac{a(a-1)}{2} f(-x) \\
& -\frac{b(b-1)}{2} f(-y)-\frac{c(c-1)}{2} f(-z)=0
\end{aligned}
$$

with nonzero real constants $a, b$, and $c$. The mapping $f(x)=$ $d x^{2}+e x$ is a solution to this functional equation, where $d, e$ are real constants. For the case $a=b=c$, the stability of the functional equation (3) was investigated by some mathematicians (see [5] for $a=b=c=1 / 3$ ).

In this paper, we will prove that if $a, b$, and $c$ are nonzero real constants, then every solution to the functional equation (3) is a quadratic-additive mapping and, conversely, we will also prove that every quadratic-additive mapping is a solution to (3) provided $a, b$, and $c$ are rational constants. Moreover, 
we will prove the generalized Hyers-Ulam stability of the functional equation (3).

We remark here that (3) is a special form of the general linear equation, whose stability was investigated by Bahyrycz and Olko [6] via a different method from that we apply in this paper. In particular, the main result of this paper is a generalization of [6].

\section{Preliminaries}

Throughout this paper, let $V$ and $W$ be vector spaces over $\mathbb{R}$, let $X$ be a real normed space, let $Y$ be a real Banach space, and let $a, b$, and $c$ be positive real constants.

For a given mapping $f: V \rightarrow W$, we use the following abbreviations:

$$
\begin{aligned}
& f_{o}(x):=\frac{f(x)-f(-x)}{2}, \\
& f_{e}(x):=\frac{f(x)+f(-x)}{2}, \\
& A f(x, y):=f(x+y)-f(x)-f(y), \\
& Q f(x, y):=f(x+y)+f(x-y)-2 f(x) \\
& -2 f(y) \text {, } \\
& D_{a, b} f(x, y):=f(a x+b y)-a b f(x+y) \\
& -a\left(\frac{a+1}{2}-b\right) f(x) \\
& -b\left(\frac{b+1}{2}-a\right) f(y) \\
& -\frac{a(a-1)}{2} f(-x) \\
& -\frac{b(b-1)}{2} f(-y) \text {, } \\
& C_{a, b} f(x, y):=f(a x+b y)+a b f(x-y) \\
& -\frac{a(2 b+a+1)}{2} f(x) \\
& -\frac{b(b+1)}{2} f(y) \\
& -\frac{a(a-1)}{2} f(-x) \\
& -\frac{b(2 a+b-1)}{2} f(-y) \text {, } \\
& D_{a, b, c} f(x, y, z):=f(a x+b y+c z)-a b f(x+y) \\
& -b c f(y+z)-a c f(x+z) \\
& -a\left(\frac{a+1}{2}-b-c\right) f(x)
\end{aligned}
$$

$$
\begin{aligned}
& -b\left(\frac{b+1}{2}-a-c\right) f(y) \\
& -c\left(\frac{c+1}{2}-a-b\right) f(z) \\
& -\frac{a(a-1)}{2} f(-x) \\
& -\frac{b(b-1)}{2} f(-y) \\
& -\frac{c(c-1)}{2} f(-z)
\end{aligned}
$$

for all $x, y, z \in V$.

The following lemmas were proved in [7].

Lemma 1. Let $a, b$ be real numbers with $(a b+1)(a+b) a b\left(a^{2}+\right.$ $\left.b^{2}+a b-1\right) \neq 0$. If a mapping $f: V \rightarrow W$ satisfies the functional equation $C_{a, b} f(x, y)=0$ for all $x, y \in V$, then $f_{e}$ is quadratic and $f_{o}$ is additive.

Lemma 2. Let $a, b$ be rational constants with $(a b+1)(a+$ b) $a b\left(a^{2}+b^{2}+a b-1\right) \neq 0$. A mapping $f: V \rightarrow W$ satisfies $C_{a, b} f(x, y)=0$ for all $x, y \in V$ if and only if $f_{e}$ is quadratic and $f_{o}$ is additive.

Since the equality

$$
C_{a, b} f(x, y)=D_{a,-b} f(x,-y)
$$

holds for all $x, y \in V$, the next couple of lemmas are direct consequences of Lemmas 1 and 2.

Lemma 3. Let $a, b$ be real numbers with $(a b-1)(a-b) a b\left(a^{2}+\right.$ $\left.b^{2}-a b-1\right) \neq 0$. If a mapping $f: V \rightarrow W$ satisfies the functional equation $D_{a, b} f(x, y)=0$ for all $x, y \in V$, then $f_{e}$ is quadratic and $f_{o}$ is additive.

Lemma 4. Let $a, b$ be rational constants with $(a b-1)(a-$ b) $a b\left(a^{2}+b^{2}-a b-1\right) \neq 0$. A mapping $f: V \rightarrow W$ satisfies $D_{a, b} f(x, y)=0$ for all $x, y \in V$ if and only if $f_{e}$ is quadratic and $f_{o}$ is additive.

Lemma 5. Let $a, b$ be rational constants. If $f: V \rightarrow W$ is a quadratic-additive mapping, then $f$ is a solution to the functional equation $D_{a, b} f(x, y)=0$ for all $x, y \in V$.

Proof. Let $f: V \rightarrow W$ be a quadratic-additive mapping. Then, $f_{e}$ is a quadratic mapping and $f_{o}$ is an additive mapping. Hence, we have $f_{e}(r x)=r^{2} f_{e}(x), f_{o}(r x)=r f_{o}(x)$, and $f_{o}(x+y)=f_{o}(x)+f_{o}(y)$, where $r$ is an arbitrary rational number.

First, we will prove that the mapping $f_{e}$ satisfies $D_{a, b} f_{e}(x, y)=0$ for arbitrary rational numbers $a$ and $b$. If $a=0$ or $b=0$, then the equality

$$
D_{a, b} f_{e}(x, y)=0
$$


holds for all $x, y \in V$. When $a=b$, the equality $D_{a, a} f_{e}(x, y)=$ 0 follows from the equality

$$
D_{a, a} f_{e}(x, y)=f_{e}(a(x+y))-a^{2} f_{e}(x+y)
$$

for all $x, y \in V$. If $a$ and $b$ are nonzero rational constants with $a \neq b$, then there exists an integer $k$ satisfying $(a k-b k)(a k b k-$ $1)\left((a k)^{2}+(b k)^{2}-a k b k-1\right) \neq 0$. According to Lemma 4 , the equality

$$
D_{a, b} f_{e}(x, y)=D_{a k, b k} f_{e}\left(\frac{x}{k}, \frac{y}{k}\right)
$$

implies that $f_{e}$ satisfies $D_{a, b} f_{e}(x, y)=0$ for all $x, y \in V$. Altogether, the mapping $f_{e}$ satisfies $D_{a, b} f_{e}(x, y)=0$ for arbitrary rational numbers $a$ and $b$.

On the other hand, the equality

$$
\begin{aligned}
D_{a, b} f_{o}(x, y)= & A f_{o}(a x, b y)-a b A f_{o}(x, y)+f_{o}(a x) \\
& -a f_{o}(x)+f_{o}(b y)-b f_{o}(y)
\end{aligned}
$$

implies that $f_{o}$ satisfies $D_{a, b} f_{o}(x, y)=0$ for arbitrary rational numbers $a$ and $b$. Since the equality $D_{a, b} f(x, y)=$ $D_{a, b} f_{e}(x, y)+D_{a, b} f_{o}(x, y)$ holds for all $x, y \in V$, we conclude that the mapping $f$ satisfies $D_{a, b} f(x, y)=0$ for all $x, y \in$ $V$.

Lemma 6. If real numbers $a, b$, and $c$ satisfy $(a-b)(b-c)(c-$ a) $\neq 0$, then either $a^{2}+b^{2}-a b-1 \neq 0$, or $a^{2}+c^{2}-a c-1 \neq 0$, or $c^{2}+b^{2}-c b-1 \neq 0$.

Proof. First, assume that $a=0$ and $a, b$, and $c$ are real constants satisfying $(a-b)(b-c)(c-a) \neq 0, a^{2}+b^{2}-a b-1=0$, $a^{2}+c^{2}-a c-1=0$, and $c^{2}+b^{2}-c b-1=0$. We then obtain the equalities $b^{2}=1, c^{2}=1$, and $c b=1$, which contradict the fact $b \neq c$.

Second, assume that $a, b$, and $c$ are nonzero real constants satisfying conditions $(a-b)(b-c)(c-a) \neq 0, a^{2}+b^{2}-a b-1=0$, $a^{2}+c^{2}-a c-1=0$, and $c^{2}+b^{2}-c b-1=0$. We then obtain the equalities $a+b=c, a+c=b$, and $a=0$, which contradict the fact $a \neq 0$.

We will now prove that $f$ is a quadratic-additive mapping provided $f$ is a solution to the functional equation $D_{a, b, c} f(x, y, z)=0$ for all $x, y, z \in V$.

Remark 7. We remark that if $\sigma:\{a, b, c\} \rightarrow\{a, b, c\}$ and $\tau$ : $\{x, y, z\} \rightarrow\{x, y, z\}$ are permutations, then we have

$$
D_{a, b, c} f(x, y, z)=0
$$

$\forall x, y, z \in V$

iff $D_{\sigma(a), \sigma(b), \sigma(c)} f(\tau(x), \tau(y), \tau(z))=0$

$\forall x, y, z \in V$
Theorem 8. Let $a, b$, and $c$ be nonzero real numbers. If $a$ mapping $f: V \rightarrow W$ satisfies the functional equation $D_{a, b, c} f(x, y, z)=0$ (with $f(0)=0$, when $a^{2}+b^{2}+c^{2}-a b-$ $b c-a c=1)$, then $f$ is a quadratic-additive mapping.

Proof. In view of Remark 7, it is enough to check the following three cases: (1), (2), and (3). Notice that $f(0)=$ $D_{a, b, c} f(0,0,0) /\left(a^{2}+b^{2}+c^{2}-a b-b c-a c-1\right)=0$, when $a^{2}+b^{2}+c^{2}-a b-b c-a c-1 \neq 0$.

(1) If $a, b$, and $c$ are real constants with $(a-b)(a-c)(b-$ $c)=0$, then we can assume that $a=b$ without loss of generality by Remark 7 . In view of Lemma 1, the equality $-a c C_{1,1} f(x, y)=D_{a, a, c} f(y,-y, x)-D_{a, a, c} f(0,0, x)$ implies that $f$ is a quadratic-additive mapping. In particular, we know that every solution to $D_{a, a, c} f(x, y, z)=0$ is a quadraticadditive mapping.

(2) If $a, b$, and $c$ are real constants with $(a b-1)(a c-1)(c b-$ 1) $=0$, then we can assume that $a b=1$ without loss of generality due to Remark 7 . We can easily show the validity of the following equalities:

$$
\begin{aligned}
& f(a x)-\frac{a^{2}+a}{2} f(x)-\frac{a^{2}-a}{2} f(-x) \\
& \quad=D_{a, 1 / a, c} f(x, 0,0), \\
& f\left(\frac{y}{a}\right)-\frac{1+a}{2 a^{2}} f(y)-\frac{1-a}{2 a^{2}} f(-y) \\
& \quad=D_{a, 1 / a, c} f(0, y, 0), \\
& f\left(\frac{z}{c}\right)-\frac{1+c}{2 c^{2}} f(c)-\frac{1-c}{2 c^{2}} f(-z)=D_{a, 1 / a, c} f(0,0, z)
\end{aligned}
$$

for all $x, y, z \in V$. Since every solution to $D_{a, a, c} f(x, y, z)=0$ is a quadratic-additive mapping, the equality

$$
\begin{aligned}
D_{1,1,1} f(x, y, z)= & D_{a, 1 / a, c} f\left(\frac{x}{a}, a y, \frac{z}{c}\right) \\
& -D_{a, 1 / a, c} f\left(\frac{x}{a}, a y, 0\right) \\
& -D_{a, 1 / a, c} f\left(\frac{x}{a}, 0, \frac{z}{c}\right) \\
& -D_{a, 1 / a, c} f\left(0, a y, \frac{z}{c}\right) \\
& +D_{a, 1 / a, c} f\left(\frac{x}{a}, 0,0\right) \\
& +D_{a, 1 / a, c} f\left(0,0, \frac{z}{c}\right) \\
& +D_{a, 1 / a, c} f(0, a y, 0)
\end{aligned}
$$

implies that $f$ is a quadratic-additive mapping.

(3) By Lemma 6 , we conclude that if $a, b$, and $c$ are real constants with $(a-b)(b-c)(c-a)(a b-1)(a c-1)(c b-1) \neq 0$, then either $(a-b)(a b-1)\left(a^{2}+b^{2}-a b-1\right) \neq 0$, or $(a-c)(a c-$ 1) $\left(a^{2}+c^{2}-a c-1\right) \neq 0$, or $(b-c)(b c-1)\left(c^{2}+b^{2}-c b-1\right) \neq 0$. By Remark 7, we can assume that $(a-b)(a b-1)\left(a^{2}+b^{2}-a b-\right.$ 1) $\neq 0$ without loss of generality. On account of Lemma 3 , the 
equality $D_{a, b} f(x, y)=D_{a, b, c} f(x, y, 0)=0$ implies that $f$ is a quadratic-additive mapping.

Theorem 9. Let $a, b$, and $c$ be rational numbers. If $f: V \rightarrow W$ is a quadratic-additive mapping, then $f$ satisfies the functional equation $D_{a, b, c} f(x, y, z)=0$.

Proof. Let $f: V \rightarrow W$ be a quadratic-additive mapping. Then we easily show that $f_{e}$ is a quadratic mapping and $f_{o}$ is an additive mapping. Hence, we have $f_{e}(r x)=r^{2} f_{e}(x)$, $f_{o}(r x)=r f_{o}(x)$, and $f_{o}(x+y)=f_{o}(x)+f_{o}(y)$, where $r$ is an arbitrary rational number. In view of Lemma 5 , we see that the mappings $f_{e}$ and $f_{o}$ satisfy the equalities $D_{a, b} f_{e}(x, y)=0$ and $D_{a, b} f_{o}(x, y)=0$. Therefore, the equalities

$$
\begin{aligned}
D_{a, b, c} f_{e}(x, y, z)= & Q f_{e}\left(a x+\frac{c z}{2}, b y+\frac{c z}{2}\right) \\
& -Q f_{e}\left(a x+\frac{c z}{2}, \frac{c z}{2}\right) \\
& -Q f_{e}\left(b y+\frac{c z}{2}, \frac{c z}{2}\right) \\
& -Q f_{e}(a x, b y)+D_{a, b} f_{e}(x, y) \\
& +D_{a, c} f_{e}(x, z)+D_{b, c} f_{e}(y, z) \\
& -f_{e}(a x)+a^{2} f_{e}(x)-f_{e}(b y) \\
& +b^{2} f_{e}(y)-4 f_{e}\left(\frac{c z}{2}\right) \\
& +c^{2} f_{e}(z), \\
& A f_{o}(a x+b y, c z)+A f_{o}(a x, b y) \\
& -a b A f_{o}(x, y)-b c A f_{o}(y, z) \\
& -a c A f_{o}(x, z)+f_{o}(a x) \\
& -a f_{o}(x)+f_{o}(b y)-b f_{o}(y) \\
& +f_{o}(c z)-c f_{o}(z) \\
D_{a, b, c}(x, y, z) & -a x)
\end{aligned}
$$

imply that the equalities $D_{a, b, c} f_{e}(x, y, z)=0$ and $D_{a, b, c} f_{o}(x, y, z)=0$ hold for all $x, y, z \in V$. From the equality $D_{a, b, c} f(x, y, z)=D_{a, b, c} f_{e}(x, y, z)+D_{a, b, c} f_{o}(x, y, z)$ for all $x, y, z \in V$, we get the equality $D_{a, b, c} f(x, y, z)=0$, as desired. and 9.

The next theorem is a direct consequence of Theorems 8

Theorem 10. Let $a, b$, and $c$ be nonzero rational constants. A mapping $f: V \rightarrow W$ satisfies $D_{a, b, c} f(x, y, z)=0$ for all $x, y, z \in V$ if and only if $f$ is a quadratic-additive mapping.

\section{Main Results}

In the following theorems, Theorems 3.1-3.3 of [8] can be slightly modified for the case when $n=3$ and $D f(x, y, z)=$ $D_{a, b, c} f(x, y, z)$ without altering their proofs.
Theorem 11. Given a real number $k$ with $|k| \neq 1$, let $\mu: V \backslash$ $\{0\} \rightarrow[0, \infty)$ be a function satisfying the condition

$$
\begin{aligned}
& \sum_{i=0}^{\infty} \frac{\mu\left(k^{i} x\right)}{k^{i}}<\infty \quad \text { when }|k|>1, \\
& \sum_{i=0}^{\infty} \frac{\mu\left(k^{i} x\right)}{k^{2 i}}<\infty \quad \text { when }|k|<1
\end{aligned}
$$

for all $x \in V \backslash\{0\}$ and let $\varphi:(V \backslash\{0\})^{3} \rightarrow[0, \infty)$ be a function satisfying the condition

$$
\begin{aligned}
& \sum_{i=0}^{\infty} \frac{\varphi\left(k^{i} x, k^{i} y, k^{i} z\right)}{|k|^{i}}<\infty \quad \text { when }|k|>1, \\
& \sum_{i=0}^{\infty} \frac{\varphi\left(k^{i} x, k^{i} y, k^{i} z\right)}{k^{2 i}}<\infty \quad \text { when }|k|<1
\end{aligned}
$$

for all $x, y, z \in V \backslash\{0\}$. If a mapping $f: V \rightarrow Y$ satisfies $f(0)=0$ and

$$
\left\|f(k x)-\frac{k^{2}+k}{2} f(x)-\frac{k^{2}-k}{2} f(-x)\right\| \leq \mu(x)
$$

for all $x \in V \backslash\{0\}$ and if $f$ satisfies

$$
\left\|D_{a, b, c} f(x, y, z)\right\| \leq \varphi(x, y, z)
$$

for all $x, y, z \in V \backslash\{0\}$, then there exists a unique mapping $F: V \rightarrow Y$ such that

$$
D_{a, b, c} F(x, y, z)=0
$$

holds for all $x, y, z \in V \backslash\{0\}$,

$$
\begin{aligned}
& F_{e}(k x)=k^{2} F_{e}(x), \\
& F_{o}(k x)=k F_{o}(x)
\end{aligned}
$$

hold for all $x \in V$, and

$$
\begin{aligned}
& \|f(x)-F(x)\| \\
& \quad \leq \sum_{i=0}^{\infty} \frac{\left|k^{i+1}+1\right| \mu\left(k^{i} x\right)+\left|k^{i+1}-1\right| \mu\left(-k^{i} x\right)}{2 k^{2 i+2}}
\end{aligned}
$$

holds for all $x \in V \backslash\{0\}$. 
Theorem 12. Given a real constant $k$ with $|k| \neq 1$, let $\mu: V \backslash$ $\{0\} \rightarrow[0, \infty)$ be a function satisfying the condition

$$
\begin{gathered}
\sum_{i=0}^{\infty} k^{2 i} \mu\left(\frac{x}{k^{i}}\right)<\infty \quad \text { when }|k|>1, \\
\sum_{i=0}^{\infty}|k|^{i} \mu\left(\frac{x}{k^{i}}\right)<\infty \quad \text { when }|k|<1
\end{gathered}
$$

for all $x \in V \backslash\{0\}$ and let $\varphi:(V \backslash\{0\})^{3} \rightarrow[0, \infty)$ be a function satisfying the condition

$$
\begin{gathered}
\sum_{i=0}^{\infty} k^{2 i} \varphi\left(\frac{x}{k^{i}}, \frac{y}{k^{i}}, \frac{z}{k^{i}}\right)<\infty \quad \text { when }|k|>1, \\
\sum_{i=0}^{\infty}|k|^{i} \varphi\left(\frac{x}{k^{i}}, \frac{y}{k^{i}}, \frac{z}{k^{i}}\right)<\infty \quad \text { when }|k|<1
\end{gathered}
$$

for all $x, y, z \in V \backslash\{0\}$. If a mapping $f: V \rightarrow Y$ satisfies $f(0)=0$ and (16) for all $x \in V \backslash\{0\}$ and if $f$ satisfies (17) for all $x, y, z \in V \backslash\{0\}$, then there exists a unique mapping $F: V \rightarrow Y$ satisfying (18) for all $x, y, z \in V \backslash\{0\}$ and (19) for all $x \in V$ and satisfying

$$
\begin{aligned}
& \|f(x)-F(x)\| \\
& \quad \leq \sum_{i=0}^{\infty}\left(\frac{\left|k^{2 i}+k^{i}\right|}{2} \mu\left(\frac{x}{k^{i+1}}\right)+\frac{\left|k^{2 i}-k^{i}\right|}{2} \mu\left(\frac{-x}{k^{i+1}}\right)\right)
\end{aligned}
$$

for all $x \in V \backslash\{0\}$.

Theorem 13. Given a real constant $k$ with $|k| \neq 1$, let $\mu: V \backslash$ $\{0\} \rightarrow[0, \infty)$ be a function satisfying the conditions

$$
\begin{array}{r}
\sum_{i=0}^{\infty} \frac{\mu\left(k^{i} x\right)}{k^{2 i}}<\infty, \\
\sum_{i=0}^{\infty}|k|^{i} \mu\left(\frac{x}{k^{i}}\right)<\infty
\end{array}
$$

$$
\text { when }|k|>1 \text {, }
$$

$$
\begin{gathered}
\sum_{i=0}^{\infty} \frac{\mu\left(k^{i} x\right)}{|k|^{i}}<\infty, \\
\sum_{i=0}^{\infty} k^{2 i} \mu\left(\frac{x}{k^{i}}\right)<\infty
\end{gathered}
$$

when $|k|<1$

for all $x \in V \backslash\{0\}$ and let $\varphi:(V \backslash\{0\})^{3} \rightarrow[0, \infty)$ be a function satisfying the conditions

$$
\begin{gathered}
\sum_{i=0}^{\infty} \frac{\varphi\left(k^{i} x, k^{i} y, k^{i} z\right)}{k^{2 i}}<\infty, \\
\sum_{i=0}^{\infty}|k|^{i} \varphi\left(\frac{x}{k^{i}}, \frac{y}{k^{i}}, \frac{z}{k^{i}}\right)<\infty
\end{gathered}
$$

when $|k|>1$,

$$
\begin{aligned}
& \sum_{i=0}^{\infty} \frac{\varphi\left(k^{i} x, k^{i} y, k^{i} z\right)}{|k|^{i}}<\infty, \\
& \sum_{i=0}^{\infty} k^{2 i} \varphi\left(\frac{x}{k^{i}}, \frac{y}{k^{i}}, \frac{z}{k^{i}}\right)<\infty
\end{aligned}
$$

when $|k|<1$

for all $x, y, z \in V \backslash\{0\}$. If a mapping $f: V \rightarrow Y$ satisfies $f(0)=0$ and (16) for all $x \in V \backslash\{0\}$ and if $f$ satisfies (17) for all $x, y, z \in V \backslash\{0\}$, then there exists a unique mapping $F: V \rightarrow Y$ satisfying (18) for all $x, y, z \in V \backslash\{0\}$ and the equalities in (19) for all $x \in V$, such that

$$
\|f(x)-F(x)\| \leq \begin{cases}\sum_{i=0}^{\infty}\left[\frac{\mu\left(k^{i} x\right)+\mu\left(-k^{i} x\right)}{2 k^{2 i+2}}+\frac{|k|^{i}}{2}\left(\mu\left(\frac{x}{k^{i+1}}\right)+\mu\left(\frac{-x}{k^{i+1}}\right)\right)\right] & \text { when }|k|>1, \\ \sum_{i=0}^{\infty}\left[\frac{k^{2 i}}{2}\left(\mu\left(\frac{x}{k^{i+1}}\right)+\mu\left(\frac{-x}{k^{i+1}}\right)\right)+\frac{\mu\left(k^{i} x\right)+\mu\left(-k^{i} x\right)}{2|k|^{i+1}}\right] & \text { when }|k|<1\end{cases}
$$

holds for all $x \in V \backslash\{0\}$.

It is to be noted that we can replace $V \backslash\{0\}$ with $V$ in Theorems 11-13.

Lemma 14. Let $a, b$, and $c$ be real constants. If $a$ mapping $f$ : $V \rightarrow W$ satisfies $f(0)=0$ and $D_{a, b, c} f(x, y, z)=0$ for all $x, y, z \in V$, then (i) $f_{e}(a x)=a^{2} f_{e}(x)$,

(ii) $f_{e}(b x)=b^{2} f_{e}(x)$,

(iii) $f_{e}(c x)=c^{2} f_{e}(x)$,

(iv) $f_{e}((a-b) x)=(a-b)^{2} f_{e}(x)$,

(v) $f_{e}((a-c) x)=(a-c)^{2} f_{e}(x)$, 
(vi) $f_{e}((b-c) x)=(b-c)^{2} f_{e}(x)$,

(vii) $f_{o}(a x)=a f_{o}(x)$,

(viii) $f_{o}(b x)=b f_{o}(x)$,

(ix) $f_{o}(c x)=c f_{o}(x)$,

(x) $f_{e}((a-b) x)=(a-b)^{2} f_{e}(x)$,

(xi) $f_{e}((a-c) x)=(a-c)^{2} f_{e}(x)$,

(xii) $f_{e}((b-c) x)=(b-c)^{2} f_{e}(x)$

for all $x \in V$.

Proof. Using the equalities

$$
\begin{aligned}
& f_{e}(a x)-a^{2} f_{e}(x)=D_{a, b, c} f_{e}(x, 0,0)=0, \\
& f_{o}(a x)-a f_{o}(x)=D_{a, b, c} f_{o}(x, 0,0)=0 \\
& f_{e}((a-b) x)-(a-b)^{2} f_{e}(x)=D_{a, b, c} f_{e}(x,-x, 0) \\
& \quad=0 \\
& f_{o}((a-b) x)-(a-b) f_{o}(x)=D_{a, b, c} f_{o}(x,-x, 0) \\
& \quad=0
\end{aligned}
$$

for all $x \in V$ and by Remark 7, we can easily prove the assertions.

Since every solution to the functional equation (3) is a quadratic-additive mapping according to Theorem 8 provided $a, b$, and $c$ are nonzero real constants, we can prove the following set of theorems by using Theorems $11-13$.

Theorem 15. For nonzero real constants $a, b$, and $c$ with $a-b \notin$ $\{-1,0,1\}$, let $\varphi: V^{3} \rightarrow[0, \infty)$ be a function satisfying the condition

$$
\sum_{i=0}^{\infty} \frac{\varphi\left((a-b)^{i} x,(a-b)^{i} y,(a-b)^{i} z\right)}{|a-b|^{i}}<\infty
$$

when $|a-b|>1$,

$$
\sum_{i=0}^{\infty} \frac{\varphi\left((a-b)^{i} x,(a-b)^{i} y,(a-b)^{i} z\right)}{(a-b)^{2 i}}<\infty
$$

$$
\text { when }|a-b|<1
$$

for all $x, y, z \in V$. If a mapping $f: V \rightarrow Y$ satisfies $f(0)=0$ and

$$
\left\|D_{a, b, c} f(x, y, z)\right\| \leq \varphi(x, y, z)
$$

for all $x, y, z \in V$, then there exists a unique quadratic-additive mapping $F: V \rightarrow Y$ such that (18) holds for all $x, y, z \in V$ and

$$
\begin{aligned}
\|f(x)-F(x)\| \\
\leq \sum_{i=0}^{\infty} \frac{\left|(a-b)^{i+1}+1\right| \varphi\left((a-b)^{i} x,-(a-b)^{i} x, 0\right)}{2(a-b)^{2 i+2}} \\
\quad+\sum_{i=0}^{\infty} \frac{\left|(a-b)^{i+1}-1\right| \varphi\left(-(a-b)^{i} x,(a-b)^{i} x, 0\right)}{2(a-b)^{2 i+2}}
\end{aligned}
$$

holds for all $x \in V$.

Proof. It follows from (29) that

$$
\begin{aligned}
& \| f((a-b) x)-\frac{(a-b)^{2}+(a-b)}{2} f(x) \\
& -\frac{(a-b)^{2}-(a-b)}{2} f(-x)\|=\| D_{a, b, c} f(x,-x, 0) \| \\
& \leq \varphi(x,-x, 0)
\end{aligned}
$$

for all $x \in V$. If we put $\mu(x)=\varphi(x,-x, 0)$ and $k=a-b$, then $\mu(x)$ satisfies condition (14) and inequality (16) for all $x \in V$. In view of Theorem 11, there exists a unique mapping $F: V \rightarrow Y$ satisfying equality (18) for all $x, y, z \in V$ and the equalities in (19) for all $x \in V$, such that inequality (30) holds for all $x \in V$.

In view of Lemma 14, the equalities in (19) follow from equality (18). Hence, there exists a unique mapping $F: V \rightarrow$ $Y$ satisfying equality (18) for all $x, y, z \in V$ and inequality (30) for all $x \in V$.

By the same way as in the proof of Theorem 15, we can prove the following couple of theorems. Hence, we omit their proofs.

Theorem 16. Given nonzero real constants $a, b$, and $c$ with $a-$ $b \notin\{-1,0,1\}$, let $\varphi: V^{3} \rightarrow[0, \infty)$ be a function satisfying the condition

$$
\begin{aligned}
& \sum_{i=0}^{\infty}|a-b|^{i} \varphi\left(\frac{x}{(a-b)^{i}}, \frac{y}{(a-b)^{i}}, \frac{z}{(a-b)^{i}}\right)<\infty \\
& \sum_{i=0}^{\infty}(a-b)^{2 i} \varphi\left(\frac{x}{(a-b)^{i}}, \frac{y}{(a-b)^{i}}, \frac{z}{(a-b)^{i}}\right)<\infty
\end{aligned}
$$

$$
\text { when }|a-b|>1
$$

for all $x, y, z \in V$. If a mapping $f: V \rightarrow Y$ satisfies $f(0)=0$ and inequality (29) for all $x, y, z \in V$, then there exists a unique quadratic-additive mapping $F: V \rightarrow Y$ satisfying (18) for all $x, y, z \in V$ and

$$
\begin{gathered}
\|f(x)-F(x)\| \leq \sum_{i=0}^{\infty}\left(\frac{\left|(a-b)^{2 i}+(a-b)^{i}\right|}{2}\right. \\
\cdot \varphi\left(\frac{x}{(a-b)^{i+1}}, \frac{-x}{(a-b)^{i+1}}, 0\right)
\end{gathered}
$$




$$
\begin{aligned}
& +\frac{\left|(a-b)^{2 i}-(a-b)^{i}\right|}{2} \\
& \left.\cdot \varphi\left(\frac{-x}{(a-b)^{i+1}}, \frac{x}{(a-b)^{i+1}}, 0\right)\right)
\end{aligned}
$$

for all $x \in V$.

Theorem 17. Given nonzero real constants $a, b$, and $c$ satisfying $a-b \notin\{-1,0,1\}$, let $\varphi: V^{3} \rightarrow[0, \infty)$ be a function satisfying the conditions

$$
\sum_{i=0}^{\infty} \frac{\varphi\left((a-b)^{i} x,(a-b)^{i} y,(a-b)^{i} z\right)}{(a-b)^{2 i}}<\infty
$$

$$
\begin{gathered}
\sum_{i=0}^{\infty}|a-b|^{i} \varphi\left(\frac{x}{(a-b)^{i}}, \frac{y}{(a-b)^{i}}, \frac{z}{(a-b)^{i}}\right)<\infty \\
\text { when }|a-b|>1, \\
\sum_{i=0}^{\infty} \frac{\varphi\left((a-b)^{i} x,(a-b)^{i} y,(a-b)^{i} z\right)}{|a-b|^{i}}<\infty, \\
\sum_{i=0}^{\infty}|a-b|^{2 i} \varphi\left(\frac{x}{(a-b)^{i}}, \frac{y}{(a-b)^{i}}, \frac{z}{(a-b)^{i}}\right)<\infty \\
\text { when }|a-b|<1
\end{gathered}
$$

for all $x, y, z \in V$. If a mapping $f: V \rightarrow Y$ satisfies $f(0)=0$ and equality (29) for all $x, y, z \in V$, then there exists a unique quadratic-additive mapping $F: V \rightarrow Y$ satisfying equality (18) for all $x, y, z \in V$ and

$$
\begin{aligned}
& \|f(x)-F(x)\| \\
& \leq\left\{\begin{array}{l}
\sum_{i=0}^{\infty}\left[\frac{\varphi\left((a-b)^{i} x,-(a-b)^{i} x, 0\right)+\varphi\left(-(a-b)^{i} x,(a-b)^{i} x, 0\right)}{2(a-b)^{2 i+2}}+\frac{|a-b|^{i}}{2}\left(\varphi\left(\frac{x}{(a-b)^{i+1}}, \frac{-x}{(a-b)^{i+1}}, 0\right)+\varphi\left(\frac{-x}{(a-b)^{i+1}}, \frac{x}{(a-b)^{i+1}}, 0\right)\right)\right] \text { when }|a-b|>1, \\
\sum_{i=0}^{\infty}\left[\frac{\varphi\left((a-b)^{i} x,-(a-b)^{i} x, 0\right)+\varphi\left(-(a-b)^{i} x,(a-b)^{i} x, 0\right)}{2|a-b|^{i+1}}+\frac{(a-b)^{2 i}}{2}\left(\varphi\left(\frac{x}{(a-b)^{i+1}}, \frac{-x}{(a-b)^{i+1}}, 0\right)+\varphi\left(\frac{-x}{(a-b)^{i+1}}, \frac{x}{(a-b)^{i+1}}, 0\right)\right)\right] \text { when }|a-b|<1
\end{array}\right.
\end{aligned}
$$

for all $x \in V$.

By the same way as in the proofs of Theorems 18-23, we can use Lemma 14 to prove Theorems 15-17.

Theorem 18. For nonzero real constants $a, b$, and $c$ with $a \notin$ $\{-1,0,1\}$, let $\varphi: V^{3} \rightarrow[0, \infty)$ be a function satisfying the condition

$$
\begin{aligned}
& \sum_{i=0}^{\infty} \frac{\varphi\left(a^{i} x, a^{i} y, a^{i} z\right)}{|a|^{i}}<\infty \quad \text { when }|a|>1, \\
& \sum_{i=0}^{\infty} \frac{\varphi\left(a^{i} x, a^{i} y, a^{i} z\right)}{a^{2 i}}<\infty \quad \text { when }|a|<1
\end{aligned}
$$

for all $x, y, z \in V$. If a mapping $f: V \rightarrow Y$ satisfies $f(0)=0$ and (29) for all $x, y, z \in V$, then there exists a unique quadratic-additive mapping $F: V \rightarrow Y$ satisfying (18) for all $x, y, z \in V$ and

$$
\begin{aligned}
\|f(x)-F(x)\| \leq & \sum_{i=0}^{\infty} \frac{\left|a^{i+1}+1\right| \varphi\left(a^{i} x, 0,0\right)}{2 a^{2 i+2}} \\
& +\sum_{i=0}^{\infty} \frac{\left|a^{i+1}-1\right| \varphi\left(-a^{i} x, 0,0\right)}{2 a^{2 i+2}}
\end{aligned}
$$

for all $x \in V$.
Proof. If we put $\mu(x)=\varphi(x, 0,0)$ and $k=a$, then it follows from (29) that

$$
\begin{gathered}
\left\|f(a x)-\frac{a^{2}+a}{2} f(x)-\frac{a^{2}-a}{2} f(-x)\right\| \\
=\left\|D_{a, b, c} f(x, 0,0)\right\| \leq \varphi(x, 0,0)
\end{gathered}
$$

for all $x \in V$. For this case, conditions (14)-(17) in Theorem 11 are satisfied. Hence, there exists a unique mapping $F: V \rightarrow Y$ satisfying equality (18) for all $x, y, z \in V$ and the equalities in (19) for all $x \in V$ and satisfying inequality (37) for all $x \in V$. Since equality (18) implies equalities (19) in Lemma 14, there exists a unique mapping $F: V \rightarrow Y$ satisfying equality (18) for all $x, y, z \in V$ as well as inequality (37) for all $x \in V$.

Theorem 19. Given nonzero real constants $a, b$, and $c$ with $a \notin$ $\{-1,0,1\}$, assume that $\varphi: V^{3} \rightarrow[0, \infty)$ is a function satisfying the condition

$$
\begin{gathered}
\sum_{i=0}^{\infty}|a|^{i} \varphi\left(\frac{x}{a^{i}}, \frac{y}{a^{i}}, \frac{z}{a^{i}}\right)<\infty \text { when }|a|<1, \\
\sum_{i=0}^{\infty} a^{2 i} \varphi\left(\frac{x}{a^{i}}, \frac{y}{a^{i}}, \frac{z}{a^{i}}\right)<\infty \text { when }|a|>1
\end{gathered}
$$


for all $x, y, z \in V$. If a mapping $f: V \rightarrow Y$ satisfies $f(0)=0$ and equality (29) for all $x, y, z \in V$, then there exists a unique quadratic-additive mapping $F: V \rightarrow Y$ satisfying (18) for all $x, y, z \in V$ and

$$
\begin{gathered}
\|f(x)-F(x)\| \leq \sum_{i=0}^{\infty}\left(\frac{\left|a^{2 i}+a^{i}\right|}{2} \varphi\left(\frac{x}{a^{i+1}}, 0,0\right)\right. \\
\left.+\frac{\left|a^{2 i}-a^{i}\right|}{2} \varphi\left(\frac{-x}{a^{i+1}}, 0,0\right)\right)
\end{gathered}
$$

for all $x \in V$.

Theorem 20. Let $a, b$, and $c$ be nonzero real constants with $a \notin\{-1,0,1\}$. Assume that $\varphi: V^{3} \rightarrow[0, \infty)$ is a function satisfying the conditions

$$
\sum_{i=0}^{\infty} \frac{\varphi\left(a^{i} x, a^{i} y, a^{i} z\right)}{a^{2 i}}<\infty
$$

$$
\sum_{i=0}^{\infty}|a|^{i} \varphi\left(\frac{x}{a^{i}}, \frac{y}{a^{i}}, \frac{z}{a^{i}}\right)<\infty
$$

when $|a|>1$,

$$
\begin{gathered}
\sum_{i=0}^{\infty} \frac{\varphi\left(a^{i} x, a^{i} y, a^{i} z\right)}{|a|^{i}}<\infty, \\
\sum_{i=0}^{\infty}|a|^{2 i} \varphi\left(\frac{x}{a^{i}}, \frac{y}{a^{i}}, \frac{z}{a^{i}}\right)<\infty
\end{gathered}
$$

when $|a|<1$

for all $x, y, z \in V$. If a mapping $f: V \rightarrow Y$ satisfies $f(0)=0$ and equality (29) for all $x, y, z \in V$, then there exists a unique quadratic-additive mapping $F: V \rightarrow Y$ satisfying (18) for all $x, y, z \in V$ and

$$
\|f(x)-F(x)\| \leq\left\{\begin{array}{l}
\sum_{i=0}^{\infty}\left[\frac{\varphi\left(a^{i} x, 0,0\right)+\varphi\left(-a^{i} x, 0,0\right)}{2 a^{2 i+2}}+\frac{|a|^{i}}{2}\left(\varphi\left(\frac{x}{a^{i+1}}, 0,0\right)+\varphi\left(\frac{-x}{a^{i+1}}, 0,0\right)\right)\right] \text { when }|a|>1, \\
\sum_{i=0}^{\infty}\left[\frac{\varphi\left(a^{i} x, 0,0\right)+\varphi\left(-a^{i} x, 0,0\right)}{2 a^{i+1}}+\frac{a^{2 i}}{2}\left(\varphi\left(\frac{x}{a^{i+1}}, 0,0\right)+\varphi\left(\frac{-x}{a^{i+1}}, 0,0\right)\right)\right] \text { when }|a|<1
\end{array}\right.
$$

for all $x \in V$.

Since every quadratic-additive mapping is a solution to the functional equation (3) and every solution to the functional equation (3) is a quadratic-additive mapping provided $a, b$, and $c$ are nonzero rational numbers by Theorem 9 , the following set of theorems are direct consequences of Theorems 15-20.

Theorem 21. For nonzero rational constants $a, b$, and $c$, let $\varphi: V^{3} \rightarrow[0, \infty)$ be a function satisfying condition (28) for all $x, y, z \in V$. If a mapping $f: V \rightarrow Y$ satisfies $f(0)=0$ and (29) for all $x, y, z \in V$, then there exists a unique quadratic-additive mapping $F: V \rightarrow Y$ satisfying (30) for all $x \in V$.

Theorem 22. For nonzero rational constants $a, b$, and $c$, let $\varphi: V^{3} \rightarrow[0, \infty)$ be a function satisfying condition (32) for all $x, y, z \in V$. If a mapping $f: V \rightarrow Y$ satisfies $f(0)=0$ and inequality (29) for all $x, y, z \in V$, then there exists a unique quadratic-additive mapping $F: V \rightarrow Y$ satisfying (33) for all $x \in V$.

Theorem 23. Given nonzero rational constants $a, b$, and $c$, assume that $\varphi: V^{3} \rightarrow[0, \infty)$ is a function satisfying the conditions in (34) for all $x, y, z \in V$. If a mapping $f: V \rightarrow Y$ satisfies $f(0)=0$ and equality (29) for all $x, y, z \in V$, then there exists a unique quadratic-additive mapping $F: V \rightarrow Y$ satisfying (35) for all $x \in V$.

Theorem 24. Let $a, b$, and $c$ be nonzero rational constants and let $\varphi: V^{3} \rightarrow[0, \infty)$ be a function satisfying condition (36) for all $x, y, z \in V$. If a mapping $f: V \rightarrow Y$ satisfies $f(0)=0$ and (29) for all $x, y, z \in V$, then there exists a unique quadratic-additive mapping $F: V \rightarrow Y$ satisfying (37) for all $x \in V$.

Theorem 25. For nonzero rational constants $a, b$, and $c$, let $\varphi: V^{3} \rightarrow[0, \infty)$ be a function satisfying condition (39) for all $x, y, z \in V$. If a mapping $f: V \rightarrow Y$ satisfies $f(0)=0$ and equality (29) for all $x, y, z \in V$, then there exists a unique quadratic-additive mapping $F: V \rightarrow Y$ satisfying (40) for all $x \in V$.

Theorem 26. Given nonzero rational constants $a, b$, and $c$, suppose $\varphi: V^{3} \rightarrow[0, \infty)$ is a function satisfying the conditions in (41) for all $x, y, z \in V$. If a mapping $f: V \rightarrow Y$ satisfies $f(0)=0$ and equality (29) for all $x, y, z \in V$, then there exists a unique quadratic-additive mapping $F: V \rightarrow Y$ satisfying (42) for all $x \in V$.

We remember that $X$ is a real normed space and $Y$ is a real Banach space.

Corollary 27. Assume that $a, b$, and $c$ are nonzero real constants with $a-b \notin\{-1,0,1\}, p$ is a real number with 
$p \notin\{1,2\}$, and $\theta$ is a positive real number. If a mapping $f: X \rightarrow Y$ satisfies $f(0)=0$ and the inequality

$$
\left\|D_{a, b, c} f(x, y, z)\right\| \leq \theta\left(\|x\|^{p}+\|y\|^{p}+\|z\|^{p}\right)
$$

for all $x, y, z \in X$, then there exists a unique quadratic-additive mapping $F: X \rightarrow Y$ such that $D_{a, b, c} F(x, y, z)=0$ holds for all $x, y, z \in X$ and

$$
\|f(x)-F(x)\| \leq \begin{cases}\frac{2 \theta\|x\|^{p}}{|| a-b|-| a-\left.b\right|^{p} \mid} & \text { when } p<1, \\ \frac{2 \theta\|x\|^{p}}{|| a-b|-| a-\left.b\right|^{p} \mid}+\frac{2\|x\|^{p}}{|| a-\left.b\right|^{2}-|a-b|^{p} \mid} & \text { when } 1<p<2, \\ \frac{2 \theta\|x\|^{p}}{|| a-\left.b\right|^{2}-|a-b|^{p} \mid} & \text { when } p>2\end{cases}
$$

holds for all $x \in X$.

Proof. If we put $\varphi(x, y, z):=\theta\left(\|x\|^{p}+\|y\|^{p}+\|z\|^{p}\right)$ for all $x, y, z \in X$, then $\varphi$ satisfies either the first inequality in (28) when $p<1$ and $|a-b|>1$, or the second inequality in (28) when $p>2$ and $|a-b|<1$, or the first inequality in (32) when $p>2$ and $|a-b|>1$, or the second inequality in (32) when $p<1$ and $|a-b|<1$, or the first inequality in (34) when $1<p<2$ and $|a-b|>1$, or the second inequality in (34) when $1<p<2$ and $|a-b|<1$ for all $x, y, z \in X$. Therefore, by Theorems 15-17, we obtain the desired inequality (44).

The following corollary follows from Theorems 18-20.

Corollary 28. Assume that $a, b$, and $c$ are nonzero real constants with $a \notin\{-1,0,1\}, p$ is a real number with $p \notin\{1,2\}$, and $\theta$ is a positive real number. If a mapping $f: X \rightarrow Y$ satisfies $f(0)=0$ and inequality (43) for all $x, y, z \in X$, then there exists a unique quadratic-additive mapping $F: X \rightarrow Y$ such that $D_{a, b, z} F(x, y, z)=0$ holds for all $x, y, z \in X$ and

$$
\begin{aligned}
& \|f(x)-F(x)\| \\
& \quad \leq \begin{cases}\frac{\theta\|x\|^{p}}{\left.|| a|-| a\right|^{p} \mid} & \text { when } p<1, \\
\frac{\theta\|x\|^{p}}{\left.|| a|-| a\right|^{p} \mid}+\frac{\|x\|^{p}}{\left.|| a\right|^{2}-|a|^{p} \mid} & \text { when } 1<p<2, \\
\frac{\theta\|x\|^{p}}{\left.|| a\right|^{2}-|a|^{p} \mid} & \text { when } p>2\end{cases}
\end{aligned}
$$

holds for all $x \in X$.

Proof. If we put $\varphi(x, y, z):=\theta\left(\|x\|^{p}+\|y\|^{p}+\|z\|^{p}\right)$ for all $x, y, z \in X$, then $\varphi$ satisfies either the first inequality in (36) when $p<1$ and $|a|>1$, or the second inequality in (36) when $p>2$ and $|a|<1$, or the first inequality in (39) when $p>2$ and $|a|>1$, or the second inequality in (39) when $p<1$ and $|a|<1$, or the first inequality in (41) when $1<p<2$ and $|a|>1$, or the second inequality in (41) when $1<p<2$ and $|a|<1$ for all $x, y \in X$. Therefore, by Theorems 18-20, we obtain the desired inequality (45).

The following corollary follows from Theorems 21-23.
Corollary 29. Let $a, b$, and $c$ be nonzero rational constants with $a-b \notin\{-1,0,1\}$ and let $\theta, p$ be nonnegative real numbers with $p \notin\{1,2\}$. If a mapping $f: X \rightarrow Y$ satisfies $f(0)=0$ and inequality (43) for all $x, y, z \in X$, then there exists a unique quadratic-additive mapping $F: X \rightarrow Y$ such that inequality (44) holds for all $x \in X$.

The following corollary follows from Theorems 24-26.

Corollary 30. Let $a, b$, and $c$ be nonzero rational constants with $a \notin\{-1,0,1\}$ and let $\theta, p$ be nonnegative real numbers with $p \notin\{1,2\}$. If a mapping $f: X \rightarrow Y$ satisfies $f(0)=0$ and inequality (43) for all $x, y, z \in X$, then there exists a unique quadratic-additive mapping $F: X \rightarrow Y$ such that inequality (45) holds for all $x \in X$.

\section{Competing Interests}

The authors declare that there is no conflict of interests regarding the publication of this paper.

\section{Authors' Contributions}

All authors contributed equally to the writing of this paper. All authors read and approved the final paper.

\section{Acknowledgments}

Soon-Mo Jung was supported by Basic Science Research Program through the National Research Foundation of Korea (NRF) funded by the Ministry of Education (no. 2015R1D1A1A02061826).

\section{References}

[1] S. M. Ulam, Problems in Modern Mathematics, John Wiley \& Sons, New York, NY, USA, 1964.

[2] D. H. Hyers, "On the stability of the linear functional equation," Proceedings of the National Academy of Sciences of the United States of America, vol. 27, pp. 222-224, 1941.

[3] T. M. Rassias, "On the stability of the linear mapping in Banach spaces," Proceedings of the American Mathematical Society, vol. 72, no. 2, pp. 297-300, 1978. 
[4] P. Găvruta, "A generalization of the Hyers-Ulam-Rassias stability of approximately additive mappings," Journal of Mathematical Analysis and Applications, vol. 184, no. 3, pp. 431-436, 1994.

[5] Y.-H. Lee and S.-M. Jung, "Generalized Hyers-Ulam stability of a 3-dimensional quadratic-additive type functional equation," International Journal of Mathematical Analysis, vol. 9, no. 9-12, pp. 527-540, 2015.

[6] A. Bahyrycz and J. Olko, "On stability of the general linear equation," Aequationes Mathematicae, vol. 89, no. 6, pp. 14611474, 2015.

[7] Y.-H. Lee and S.-M. Jung, "Stability of some 2-dimensional functional equations," International Journal of Mathematical Analysis, vol. 10, no. 4, pp. 171-190, 2016.

[8] Y.-H. Lee and S.-M. Jung, "A general stability theorem for a class of functional equations including quadratic-additive functional equations," Journal of Computational Analysis and Applications, In press. 


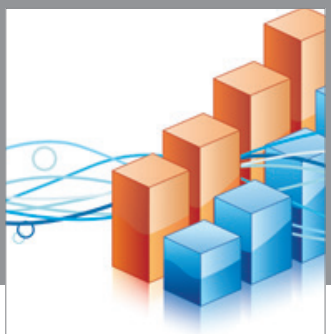

Advances in

Operations Research

vatem alat4

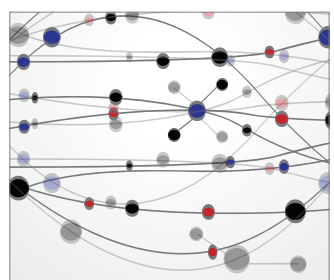

\section{The Scientific} World Journal
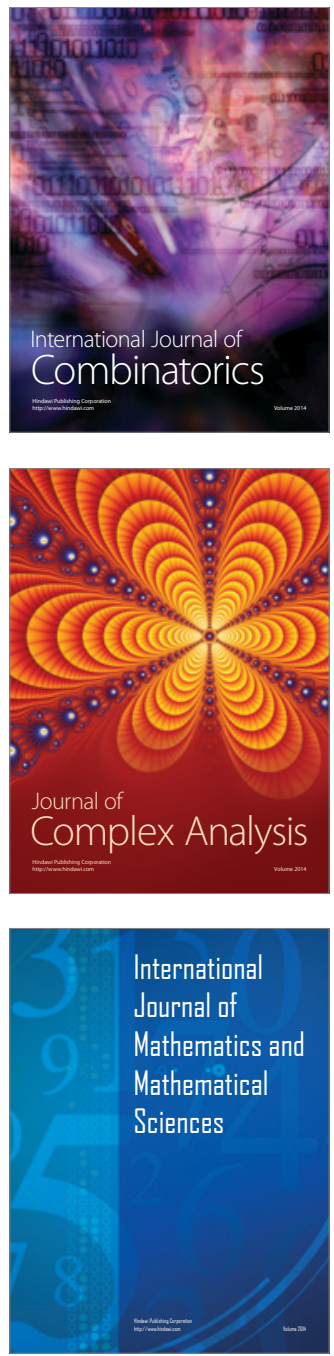
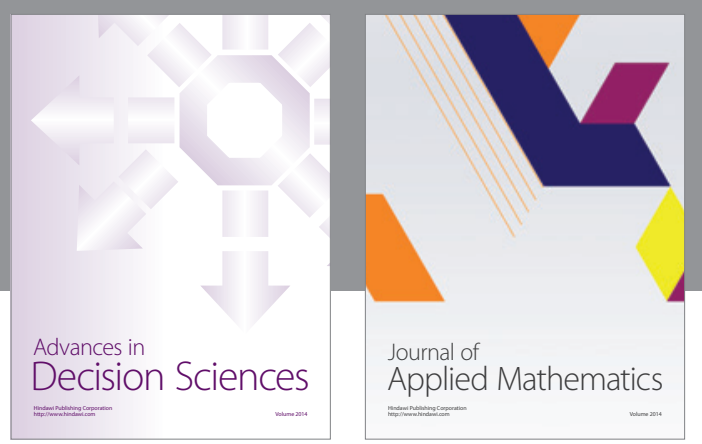

Algebra

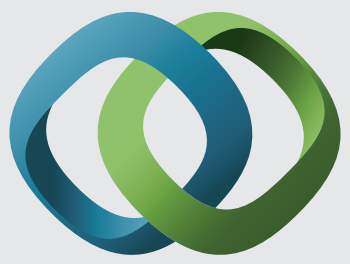

\section{Hindawi}

Submit your manuscripts at

http://www.hindawi.com
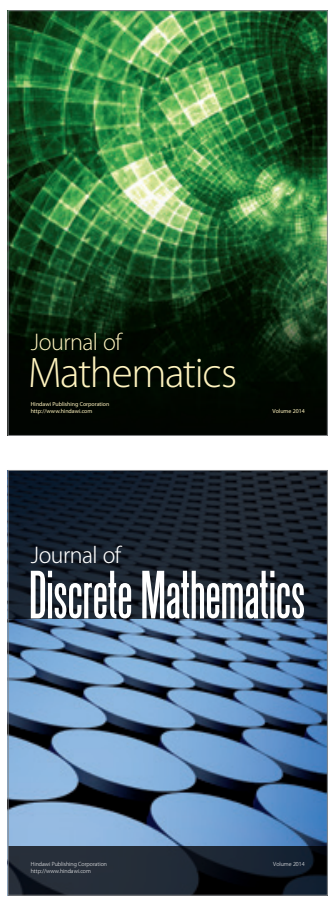

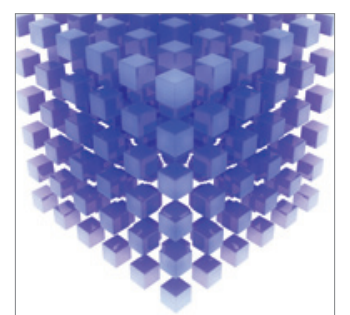

Mathematical Problems in Engineering
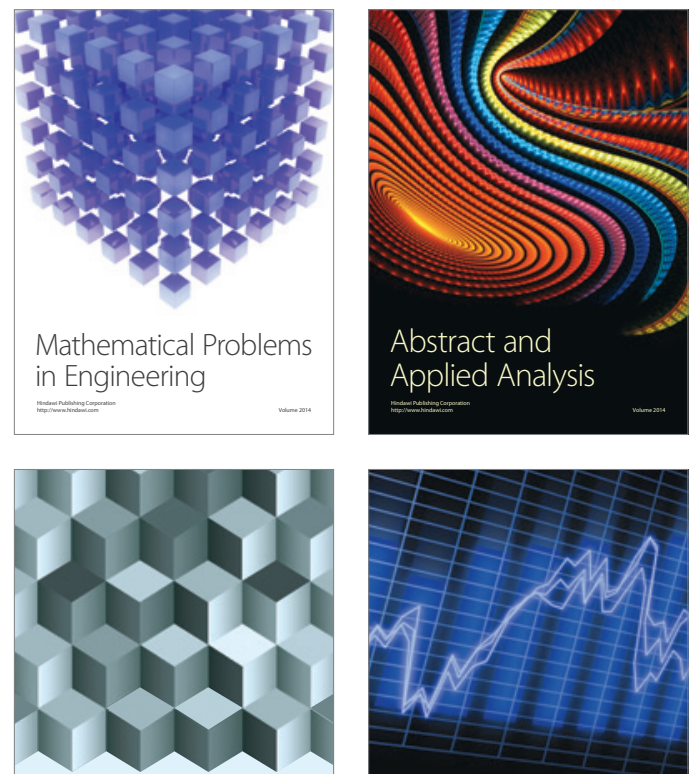

Journal of

Function Spaces

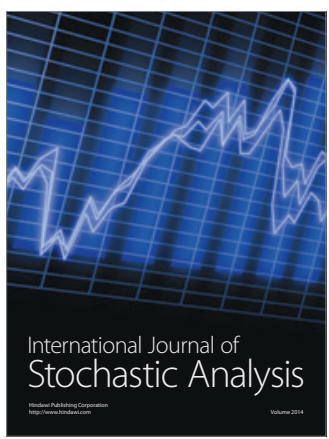

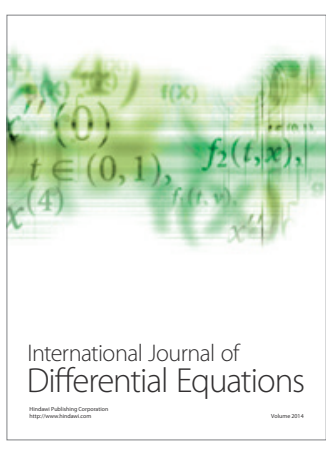
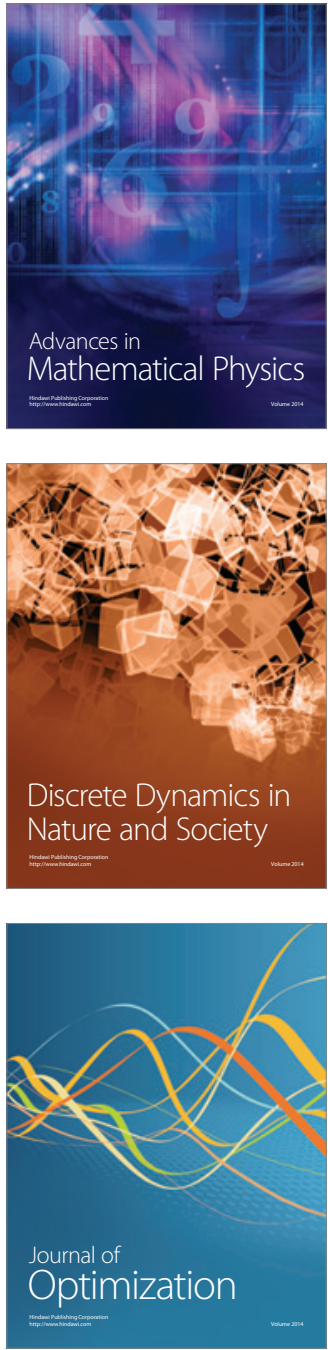